\title{
BIOFÍSICA AMBIENTAL DO SEMIÁRIDO: QUADRO PARADIDÁTICO PARA EDUCAÇÃO BÁSICA
}

\author{
Raquel Bernardo de Melo ${ }^{1 *}$, Clodoaldo de Lima ${ }^{1}$, \\ Carla Valéria de Miranda Costa Duarte ${ }^{1}$, Susana Carvalho de Souza ${ }^{1,2}$, Otacilio Antunes Santana ${ }^{1,3}$ \\ ${ }^{1}$ Mestrado Profissional em Rede Nacional para Ensino das Ciências Ambientais - PROFCIAMB, UFPE; \\ ${ }^{2}$ Departamento de Micologia, Centro de Biociências, UFPE \\ ${ }^{3}$ Departamento de Biofísica e Radiobiologia, Centro de Biociências, UFPE \\ *raquelbernardom@gmail.com
}

\section{INTRODUÇÃO}

A Educação Contextualizada é uma forma de unir a leitura do mundo, feita pelos alunos, com os conteúdos curriculares delimitados para cada ciclo educacional (FREIRE, 2014). Por exemplo, o aluno vai à escola de ônibus, e nesse percurso da casa a escola, ele poderá vivenciar fatos que poderão ajudá-lo na futura consolidação de um conhecimento. Esse conceito de conhecimento prévio, ou aprendizagem significativa prévia, é de basilar valor para a aprendizagem, em que o aluno de forma coletiva e interacional poderá por meio de suas prévias práticas, ou visão do mundo, hipotetizar sobre o conteúdo a ser ensinado e a contribuir com sua experiência a certificar a teoria (VIGOTSKY, 1991).

A aproximação dos conteúdos ao afeto (ou ao desafeto), ao vivido, é uma missão do mediador no ambiente educacional, na relação coletiva e com os objetos educacionais a serem construídos e compartilhados (TOASSA, 2011). Por isso, há uma importância na construção de recursos e situações didáticas que aproximem os conteúdos aos contextos: espaciais, temporais, sociais, culturais, dos alunos.

Os livros didáticos tentam ser genéricos para atingir ao máximo de situações contextuais, porém com essa generalidade se perde o particular e o singular do aluno (LAJOLO, 1996). Assim sem a contextualização, o aluno sente que o objeto de estudo não pertence a ele ou a seu ambiente, deixando-o distante, sem familiarização, sem um sentido simbólico. Outro destaque nos livros didáticos, é a fragmentação do conhecimento (MORIN, 2008), em que parece que variáveis físicas nunca se correlacionarão com o desenvolvimento biótico, por exemplo.

Os recursos paradidáticos vêm para suprir essa lacuna da não contextualização e da fragmentação do conhecimento (MIYAKAWA; WINSLOW, 2013; RODRIGUES, 2015). Na literatura se apresenta vá rios vieses de aplicações a justificar a importância de se trabalhar didaticamente e paradidaticamente os quadros didáticos no processo de ensino e aprendizagem, como o proposto nesse trabalho (Tabela 1).

0 objetivo desse trabalho foi apresentar um quadro paradidático que une visões interdisciplinares em um contexto: biofísica ambiental do Semiárido, baseada na educação contextualizada, no qual se terá um momento lúdico com um objeto regional e uma recomendação para uma demanda regional: potabilidade da água.

Tabela 1. Referencial Teórico e a importância no campo da produção e aplicação de Quadros Paradidáticos.

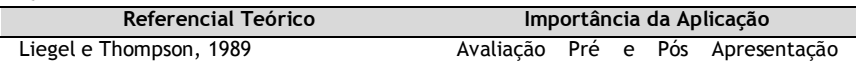

\begin{tabular}{|c|c|}
\hline & (PreDisplay e PostDisplay) \\
\hline Cigler, 1990 & Integração entre o símbolo e o oral \\
\hline Allen, 1994 & Delimitação de sequencias históricas \\
\hline Billington, 1997 & $\begin{array}{l}\text { Avaliação da aprendizagem como portfó } \\
\text { lio do aluno }\end{array}$ \\
\hline Crowley-Long, Powell e Christensen, 1997 & Exposição de pesquisa e arte dos Alunos \\
\hline Bracher; Cantrell; Wilkie, 1998 & $\begin{array}{l}\text { A vivência da exposição de um objeto } \\
\text { de aprendizagem }\end{array}$ \\
\hline Kumagai, 1998 & Simplificação Didática das Exatas \\
\hline Hay e Thomas, 1999 & $\begin{array}{l}\text { O fazer sentido da contextualização na } \\
\text { Biologia }\end{array}$ \\
\hline Akister e Bannon, 2000 & Educação no Trabalho Social \\
\hline Marek, Christopher e Koenig, 2002 & Aplicação Técnica e Tecnológica \\
\hline Middleton e Midgley, 2002 & $\begin{array}{l}\text { Motivação frente as percepções das } \\
\text { Exatas }\end{array}$ \\
\hline Mills, 2007 & Empoderamento dos alunos \\
\hline Gillespie, 2009 & $\begin{array}{l}\text { Valorização da autoria e co-autoria e o } \\
\text { trabalho cultural }\end{array}$ \\
\hline Lapp e Fisher, 2009 & $\begin{array}{l}\text { Motivação dos alunos a lerem e } \\
\text { escreverem }\end{array}$ \\
\hline White, 2010 & $\begin{array}{l}\text { Desmistificação de conteúdos, como o } \\
\text { da matemática }\end{array}$ \\
\hline Hubenthal, O’Brien e Taber, 2011 & $\begin{array}{l}\text { Promoção da cognição individual e } \\
\text { coletiva }\end{array}$ \\
\hline Faunce, 2012 & $\begin{array}{l}\text { Composição e integração dos conteúdos } \\
\text { em sala de aula: mapa conceitual }\end{array}$ \\
\hline Shearer, Snider e Kniel, 2013 & Educação da segurança alimentar \\
\hline Altintas et al., 2014 & Educação financeira doméstica \\
\hline $\begin{array}{l}\text { Scheil, Bowers-Campbell e Campbell, } \\
2017\end{array}$ & $\begin{array}{l}\text { Educação de Crianças para Crianças } \\
\text { deficientes }\end{array}$ \\
\hline
\end{tabular}

DEMANDA EDUCACIONAL E SOCIAL

A construção do quadro paradidático é uma demanda educacional, primeiro para se integralizar conhecimentos: físicos, ambientais, matemáticos, geoespaciais, entre outros e pensar o conhecimento de forma inter e transdisciplinar. A segunda demanda educacional é o estudo do contexto (contextualização). O Semiárido, clima que rege sob algumas regiões, é caracterizado por ter a falta de um regime pluviométrico definido, precipitações anuais abaixo de $500 \mathrm{~mm}$ e com balanço hídrico negativo (evapotranspiração maior que precipitação). Nas áreas em que está sob o Semiárido, a baixa ciclagem hídrica em relação a outros ecossistemas (e.g. Mata Atlâ ntica), causa uma baixa produtividade primária (e.g. atividade e produtos fotossintéticos). Essa baixa produtividade faz com que as políticas governamentais não priorizem essas regiões com planos de desenvolvimento econômico, a ocasionar locais com vulnerabilidade não só ambiental (desmatamento para lenha; SANTANA, 2016 e 2017), como também social (Índice de Vulnerabilidade Social = 0,278; IPEA, 2010).

Com pouca água, baixa produtividade e falta de reconhecimento governamental, o que restou a essas regiões foi o abandono do 
Estado, a apropriação patrimonial e ambiental por exploradores nã o locais, e o esquecimento até da literatura (livro didático). 0 Seminárido e a Caatinga são as regiões menos estudadas e documentadas educacionalmente, ambientalmente e socialmente (SAUTCHUK, 2015). Por isso, construir um quadro paradidático sobre a região é emergencial para que profissionais da educação, e líderes comunitários possam, via conhecimento, alertar a comunidade sobre a importância local e da erradicação de sua destruição como ecossistema (atuação profissional).

\section{CONSTRUÇÃO DO QUADRO PARADIDÁTICO}

o quadro paradidático foi construído no programa InkScape (InkScape, 2017), em que centralmente está uma representação visual do ambiente do Semiárido com elementos abióticos (solo) e bióticos (fauna e flora). Nesse cenário, estão elencadas as variáveis físicas que interagem com o ambiente e seus elementos, voltadas principalmente para a temática da ciclagem da água, recurso limitante da região (TEJERO; ZUAZO, 2018).

O cenário foi construído a partir de encontros prévios (Figura 1) com docentes que lecionam em escolas públicas que tem alguma relação com as regiões semiáridas. E as variáveis dispostas conforme os balanços hídricos e energéticos propostos por
Campbell e Norman (1998), para região (SANTANA, 2016). Abaixo da representação visual (Figura 2), estão os conceitos em uma linguagem simplificada em que os leitores dos livros didáticos possam compreender sem necessitar de uma compreensão hermenê utica.



Figura 1. Docentes da Educação Básica a sugerir cenários do Semiárido.

\section{Biofísica Ambiental do SemiáRIdo}

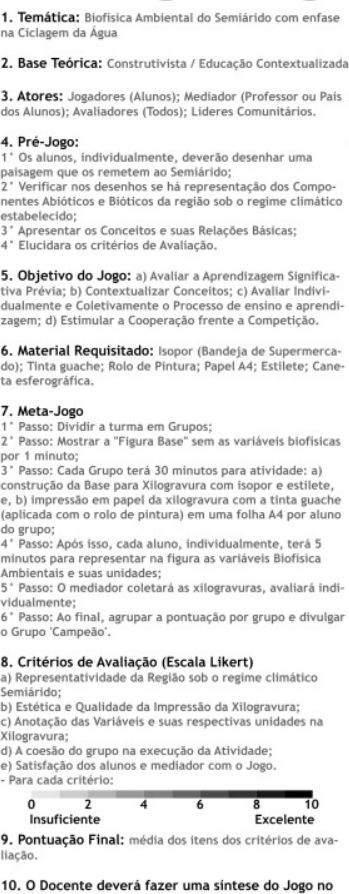
JOGO DA
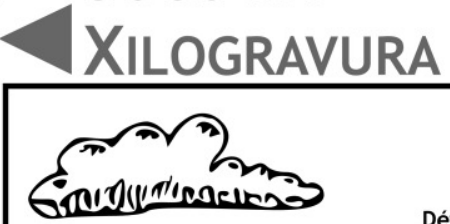

Precipitação $(\mathrm{mm})$

Vento $\left(\mathrm{m} \mathrm{s}^{-1}\right)$

Evapotranspiração $(\mathrm{mm})$

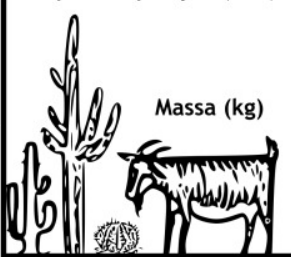

POTABILIDADE DA ÁGUA

Temperatura $\left({ }^{\circ} \mathrm{C}\right)$

Déficit de pressão de vapor de água (kPa)

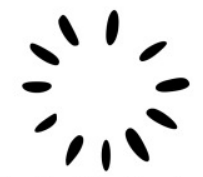

Radiação $\left(W \mathrm{~m}^{-2} \mathrm{~cm}\right)$

Fotossintese $\left(\mu \mathrm{mol} \mathrm{m} \mathrm{m}^{-2} \mathrm{~s}^{-1}\right)$

Respiração (VO2)

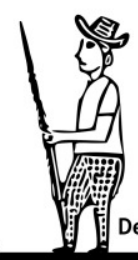

Abertura de Dossel (\%)

Densidade da Madeira $\left(\mathrm{g} \mathrm{cm}^{-3}\right)$
10. O Docente deverá fazer uma sintese do Jogo no
processo de ensino e aprendizagem

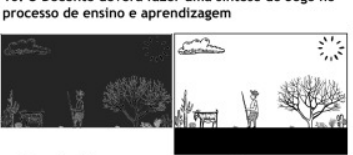

Base da Xilogravura Xilogravura

CONCEITOS
Densidade do Solo $\left(\mathrm{g} \mathrm{cm}^{-3}\right)$

$\Psi_{\mathrm{s}}(\mathrm{MPa})$



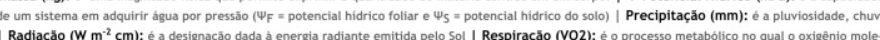

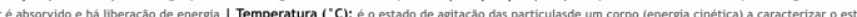

Figura 2. Quadro Paradidático Construído.

Em uma das laterais está disposta uma atividade lúdica a ser aplicada dentro e fora do ambiente de ensino e aprendizagem. Essa atividade se baseia na construção coletiva de uma base de xilogravura, que se assemelha com o cenário apresentado no quadro paradidático, em que os alunos em grupo construirão um cenário para representar seu imaginário sobre o Semiárido. Após a construção da base, as xilogravuras são impressas em papel A4, para que individualmente cada um acrescente as variáveis no cená 
rio. E ao final, todo o processo é avaliado conforme Santana e Petrova, 2016.

A xilogravura foi escolhida para o momento lúdico por sua importâ ncia histórica no imaginário do sertanejo residente do Semiárido, pois é a base de reprodução de figuras e painéis que representam momentos históricos, costumes e situações locais, como também de base para a montagem da Literatura de Cordel (CNFCP, 2017).

$\mathrm{Na}$ outra lateral, está um sistema para potabilizar água no Semiá rido, com um filtro orgânico, construído com um tecido vegetal da planta (Luffa aegyptiaca Mill. Curcubitaceae) e com uma base para concentrar a radiação solar na água, a causar desinfecção microbió tica. Esse sistema foi construído baseado na literatura (WEGELIN et al., 1994).

0 quadro didático construído está disponível no formato PDF, no repositório OERCommons (2017), sob livre acesso, com obrigatoriedade de atribuição e ser não comercial (CC, 2017), construído para um público-alvo em geral com nível escolar para estudantes do ensino médio.

\section{VALIDAÇÃO E APLICAÇÃO}

A validação do quadro paradidático foi realizada por seis docentes que realizam atividades de ensino e/ou pesquisas em escolas pú blicas da educação básica (Figura 3), por meio dos critérios de avaliação da Coordenação de Avaliação das Ciências Ambientais da Coordenação de Aperfeiçoamento de Pessoal de Nível Superior (CAPES, 2017). Os critérios são: a) Aderência: se os conteúdos apresentados estão no Livro Didático do Ensino Médio; b) Impacto: se o objeto educacional causará alguma implicação social (impacto ambiental, impacto sanitário, impacto cultural, impacto econô mico, etc...); c) Aplicabilidade: se o objeto educacional é de fácil manuseio e compreensão, se suas regras e propostas são autoexplicativas, e de utilidade para o público-alvo estabelecido; d) Inovação: se o objeto educacional rompe metodologicamente com os recursos didáticos recorrentes; e, e) Complexidade: se o objeto educacional é direcionada a uma diversidade de atores, relações e conhecimentos necessários à elaboração e ao desenvolvimento educacional (Figura 4).

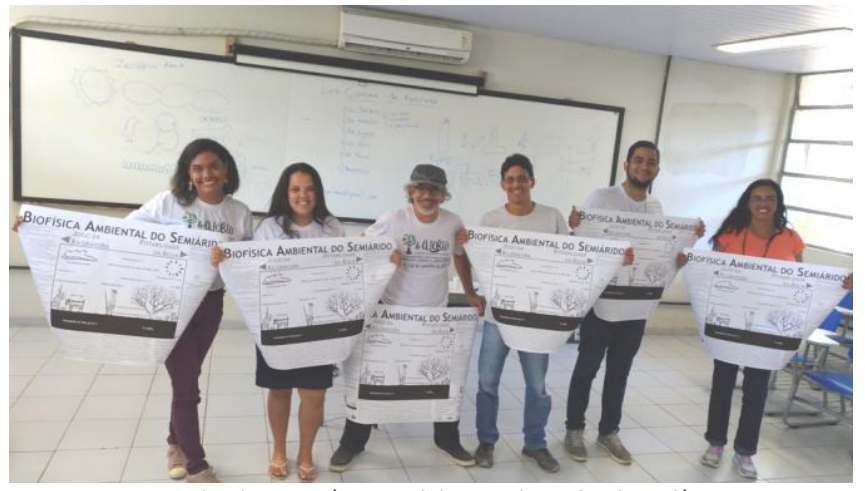

Figura 3. Docentes da Educação Básica a validar e avaliar o Quadro Didático.



Figura 4. Validação do Quadro Paradidático por meio dos critérios de avaliação da Coordenação de Avaliação das Ciências Ambientais da CAPES (Escala Likert).

A aplicação foi feita pelos docentes em suas turmas, nas quais participaram da atividade lúdica proposta pelo quadro didático 157 alunos do ensino médio. Ao final os próprios docentes avaliaram a atividade lúdica, segundo os critérios pré-estabelecidos no objeto educacional e baseado nas avaliações de Santana e Petrova (2016): a) Representatividade da Região sob o regime climático Semiárido; b) Estética e Qualidade da figura produzida; c) Anotação das Variá veis e suas respectivas unidades na Xilogravura; d) A coesão do grupo na execução da Atividade; e, e) Satisfação dos alunos e mediador com o Jogo (Figura 5).

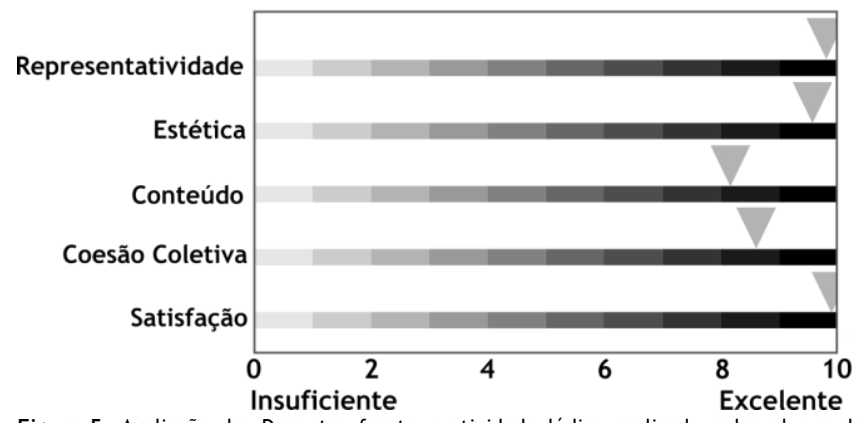

Figura 5. Avaliação dos Docentes frente a atividade lúdica realizada pelos alunos da educação básica (Escala Likert).

Tanto nos critérios de validação (aderência, impacto, aplicabilidade, complexidade e inovação) como nos da aplicação (representatividade, estética, conteúdo, coesão coletiva e satisfaç ão) receberam pesos acima de oito na escala Likert de avaliação, mostrando a efetividade e eficácia do uso do quadro paradidático construído para o objetivo proposto, ensinar a Biofísica Ambiental do Semiárido utilizando ferramentas contextualizadas (Figura 4 e 5). A satisfação do uso do quadro na atuação profissional foi outro ponto de destaque na avaliação, revelando-se eficiente não só para o conteúdo estabelecido, como também para outros conteúdos a serem trabalhados.

\section{CONSIDERAÇÕES FINAIS}

O quadro paradidático construído atingiu seu objetivo de unir visõ es interdisciplinares em um contexto: biofísica ambiental do Semiá rido, baseada na educação contextualizada. Esse recurso didático cumpriu as demandas solicitadas socialmente de ser um atributo para atuação profissional (instrumento lúdico) e por ter implicaçõ es sociais, na instrumentalização de um sistema de potabilidade da água para o Semiárido. O sucesso da validação do quadro foi certificado pelos docentes em sua aplicação, demonstrado pela adequação dos resultados aos critérios de avaliação, validação e aplicação. Os autores prospectam a divulgação do quadro construí do para outros docentes em escolas inseridas no Semiárido, aumentando o número de avaliadores, usuários e com isso novas sugestões e correções que serão inseridas ao recurso paradidático.

\section{REFERÊNCIAS}

AKISTER, J.; BANNON, A.; MULLENDER-LOCK, H. Poster Presentations in Social Work Education. Innovations in Education and Training International, v. 37, n. 3, p. 229-233, 2000.

ALLEN, R. F. Posters as Historical Documents: A Resource for the Teaching of Twentieth-Century History. The Social Studies, v. 85, n. 2, p. 52-61, 1994. DOI: 10.1080/00377996.1994.9956276

ALTINTAS, N. N.; SUER, A. Z.; SARI, E. S.; ULKER, M. S. The Use of Poster Projects as a Motivational and Learning Tool in Managerial 
Accounting Courses. Journal of Education for Business, v. 89, n. 4, p. 196-201, 2014. Doi: 10.1080/08832323.2013.840553

BILLINGTON, H. L. Poster presentations and peer assessment: novel forms of evaluation and assessment. Journal of Biological Education, v. 31, n. 3, p. 218-220, 1997. Doi: 10.1080/00219266.1997.9655566

BRACHER, L.; CANTRELL, J.; WILKIE, K. The process of poster presentation: a valuable learning experience. Medical Teacher, $v$. 20, n. 6, p. 552-557, 1998.

CAMPBELL, G. S.; NORMAN, J. M. Introduction to environmental biophysics. 2nd ed. New York: Springer-Verlag. 1998. 287p.

CAPES - Coordenação de Aperfeiçoamento de Pessoal de Nível Superior (2017). Ciências Ambientais - Área de Avaliação. Disponí vel em: https://goo.gl/FuhCDN Acesso em 25/11/2017.

CIGLER, B. A. Integrated written and oral assignments for the MPA curriculum. International Journal of Public Administration, v. 13, n. 1-2, p. 325-342, 1990. DOI: 10.1080/01900699008524676

CLARKE-SATHER, A. State power and domestic water provision in semi-arid Northwest China: Towards an aleatory political ecology. Political Geography, v. 58, p. 93-103, 2017. Doi: 10.1016/j.polgeo.2015.12.003

CNFCP - Centro Nacional de Folclore e Cultura Popular. Xiloteca. Disponível em https://goo.gl/PpT2Ej Acesso em 25/11/2017.

CC - CREATIVE COMMONS. License. Disponível em https:// creativecommons.org/ Acesso em 25/11/2017.

CROWLEY-LONG, K.; POWELL, J. L.; CHRISTENSEN, C. Teaching Students about Research: Classroom Poster Sessions. The Clearing House: A Journal of Educational Strategies, Issues and Ideas, v. 70, n. 4, p. 202-204, 1997. Doi: 10.1080/00098655.1997.10544197

FAUNCE, R. Teaching Querelle in the Composition Classroom. Pedagogy, v. 12, n. 2, p. 343-381, 2012.

FREIRE, P. Alfabetização: leitura do mundo, leitura da palavra. Editora Paz e Terra, 2014.

GILLESPIE, T. Characterizing Copyright in the Classroom: The Cultural Work of Antipiracy Campaigns. Communication, Culture \& Critique, v. 2, p. 274-318, 2009. Doi: 10.1111/j.17539137.2009.01039.x

HAY, I.; THOMAS, S. M. Making sense with posters in biological science education. Journal of Biological Education, v. 33, n. 4, p. 209-214, 1999. Doi: 10.1080/00219266.1999.9655668

HENINI, G.; LAIDANI, Y.; SOUAHI, F.; HANINI, S. Study of Static Adsorption System Phenol / Luffa Cylindrica Fiber for Industrial Treatment of Wastewater. Energy Procedia, v. 18, p. 395-403, 2012. Doi: $10.1016 /$ j.egypro.2012.05.051

HUBENTHAL, M.; O'BRIEN, T.; TABER, J. Posters that foster cognition in the classroom: multimedia theory applied to educational posters. Educational Media International, v. 48, n. 3, p. 193-207, 2011. Doi: 10.1080/09523987.2011.607322

INKSCAPE. Download. Disponível em: https: //inkscape.org/ Acesso em: 25/11/2017.

IPEA - Instituto de Pesquisas Econômicas Aplicadas. Índice de Vulnerabilidade Social 2010. Disponível em: http: //ivs.ipea.gov.br Acesso em: 25/11/2017.

KUMAGAI, J. Classroom Poster Sums Up Nuclear Science Concepts. Physics Today, v. 51, n. 11, p. 54-61, 1998. Doi: 10.1063/1.882052 LAPP, D.; FISHER, D. It's All About the Book: Motivating Teens to Read. Journal of Adolescent \& Adult Literacy, v. 52, n. 7, p. 556561, 2009. Doi: 10.1598/JAAL.52.7.1

LAJOLO, M. Livro didático: um (quase) manual de usuário. Em Aberto, v. 16, n. 69, 1996.
LIEGEL, L. H.; THOMPSON, D. Poster presentations for scientific meetings. Journal Agronomy Education, v. 18, n. 2, p. 69-75, 1989.

MAREK, P.; CHRISTOPHER, A. N.; KOENIG, C. S. Applying Technology to Facilitate Poster Presentations. Teaching of Psychology, v. 29, n. 1, p. 70-72, 2002. Doi: 10.1207/S15328023TOP2901_12

MIDDLETON, M. J.; MIDGLEY, C. Beyond Motivation: Middle School Students' Perceptions of Press for Understanding in Math. Contemporary Educational Psychology, v. 27, p. 373-391, 2002. Doi: 10.1006/ceps.2001.1101

MILLS, K. A. “Have You Seen Lord of the Rings?” Power, Pedagogy, and Discourses in a Multiliteracies Classroom. Journal of Language, Identity \& Education, v. 6, n. 3, p. 221-241, 2007. Doi: 10.1080/15348450701454247

MIYAKAWA, T.; WINSLOW, C. Developing mathematics teacher knowledge: the paradidactic infrastructure of "open lesson" in Japan. Journal of Mathematics Teacher Education, v. 16, n. 3, p. 185-209, 2013. https://doi.org/10.1007/s10857-013-9236-5 MORIN, E. A cabeça bem-feita: repensar a reforma, reformar o pensamento. 20. ed. Rio de Janeiro: Bertrand, 2012. 128 p.

OERCommons. Open Educational Resources. Disponível em: https://www.oercommons.org/ Acesso em: 25/11/2017.

RODRIGUES, M. A. A leitura e a escrita de textos paradidáticos na formação do futuro professor de Física. Ciências e Educação, v. 21, n. 3, p. 765-781, 2015. Doi: 10.1590/1516-731320150030015.

SANTANA, O. A. Resistência social na Caatinga árida: a narrativa de quem ficou no colapso ambiental. Desenvolvimento e Meio Ambiente, v. 38, p. 419-438, 2016. Doi: 10.5380/dma.v38i0.43574 SANTANA, O. A.; PETROVA, Y. Ludicidade no Ensino da Normalidade em um Ambiente Florestal. Inter-ação, v. 41, p. 525544, 2016. Doi: 10.5216/ia.v41i3.41502

SANTANA, O. A. Minimum age for clear-cutting native species with energetic potential in the Brazilian semi-arid region. Canadian Journal of Forest Research, v. 47, p. 411-417, 2017. Doi: 10.1139/cjfr-2016-0392

SAUTCHUK, J. Caatinga: Bioma desprezado sobrevive no semiárido. (2015). Disponível em https://goo.gl/vA8s37 Acesso em 25/11/2017.

SCHEIL, K. A.; BOWERS-CAMPBELL, J.; CAMPBELL, J. M. An Initial Investigation of the Kit for Kids Peer Educational Program. Journal of Developmental and Physical Disabilities, v. 29, n. 4, p. 643662, 2017. Doi: $10.1007 / \mathrm{s} 10882-017-9540-6$

SHEARER, A. E. H.; SNIDER, O. S.; KNIEL, K. E. Development, Dissemination, and Preimplementation Evaluation of Food Safety Educational Materials for Secondary Education. Journal of Food Science Education, v. 12, p. 28-37, 2013. Doi: 10.1111/15414329.12004

TEJERO, I. F. G.; ZUAZO, V. H. D. Water Scarcity and Sustainable Agriculture in Semiarid Environment. 1st Ed. Cambridge: Academic Press. 2018. 624p.

TOASSA, G. Emoções e vivências em Vigotski. 1. ed. Campinas, SP: Papirus, 2011. v. 1. 288p.

VIGOTSKY, L. S. Pensamento e linguagem. 3. ed. São Paulo, SP: Martins Fontes, 1991. 135 p.

WEGELIN, M.; CANONICA, S.; MECHSNER, K.; FLEISCHMANN, T.; PESARO, F.; METZLER, A. Solar water disinfection: scope of the process and analysis of radiation experiments. Aqua, v. 43, n. 4, p. 154-169, 1994.

WHITE, A. L. Numeracy, Literacy and Newman's Error Analysis. Journal of Science and Mathematics Education in Southeast Asia, v. 33, n. 2, p. 129-148, 2010. 